\title{
A New Low-Power Recoding Algorithm for Multiplierless Single/Multiple Constant Multiplication
}

\author{
A.K. Oudjida, M.L. Berrandjia \\ Microelectronics and Nanotechnology Division \\ Centre de Développement des Technologies Avancées \\ Algiers, Algeria \\ a_oudjida@cdta.dz
}

\author{
N. Chaillet \\ AS2M Department \\ FEMTO-ST Institute \\ Besançon, France \\ nicolas.chaillet@ffemto-st.fr
}

\begin{abstract}
Optimizing the number of additions in constant coefficient multiplication is conjectured to be a NP-hard problem. In this paper, we report a new heuristic requiring an average of $\mathbf{2 9 . 1 0 \%}$ and $\mathbf{1 0 . 6 1 \%}$ less additions than the standard canonical signed digit representation (CSD) and the double base number system (DBNS), respectively, for 64-bit coefficients. The maximum number of additions per coefficient is bounded by $(N / 4)+2$, and the time-complexity of the recoding is linearly proportional to $N$, where $N$ is the bit-size of the constant. These performances are achieved using a new redundant version of radix $-2^{8}$ recoding.
\end{abstract}

Keywords-Double Base Number System (DBNS); High-Speed and Low-Power Design; Multiplierless Single/Mutiple Constant Multiplication (SCM/MCM); Radix-2 ${ }^{r}$ Booth recoding.

\section{BACKGROUND AND MOTIVATION}

Many applications in DSP and control, such as linear time invariant (LTI) filters/controllers, involve the computation of a large number of multiplications of one variable by a set of constants. To be efficiently handled, the implementation must be multiplierless, that is, using exclusively additions, subtractions and shifts. This problem is known as single/multiple constant multiplication (SCM/MCM) and is conjectured to be NP-hard [1]. A big number of heuristics have been proposed. They are classified into four categories:

- Digit-recoding heuristics such as CSD [2] and DBNS [3] ;

- Common subexpression elimination (CSE) using pattern matching. Examples are Lefèvre [4] and Boullis [5];

- Directed acyclic graph (DAG) based algorithms such as Hcub [6], H(k) [7], and MAG [8];

- Mixed algorithms combining CSE and DAG such as the recent optimal algorithm BIGE [1].

A good survey and a detailed comparative study showing pros and cons of various algorithms is given in [1][6][9].

Despite the big number of proposed heuristics, the vast majority of LTI system optimizations use the CSD representation for constant encoding [10]. The rational is that:

- CSD recoding is easy to implement;

- The adder complexity in CSD is known, which is not the case for the other heuristics [1][11]. In CSD the number of adders is bounded by $(N+1) / 2-1$ and tends asymptotically to an average value of $(N / 3)-8 / 9$, which yields to $33 \%$ saving over the naïve add-and-shift approach.

This work is supported by "Centre de Développement des Technologies Avancées (CDTA), Algiers, Algeria, in collaboration with FEMTO-ST Institute, Besançon, France.
- CSD requires a linear computational time, contrary to its counterparts that require an excessive runtime (Table I) and storage, which makes them impractical for high values of $N$, at least for the current compute power.

TABLE I. ASSYMPTOTIC RUNTIME SUMMARY

\begin{tabular}{|c|l|c|c|}
\hline \hline \multicolumn{3}{|c|}{ Algorithm } & \multirow{2}{*}{ Runtime } \\
\cline { 1 - 3 } Name & \multicolumn{1}{|c|}{ Author } & Year & \\
\hline BIGE & Thong [1] & 2011 & $\mathrm{O}\left(2^{N}\right)$ \\
\hline H(k) & Dempster [7] & 2004 & $\mathrm{O}\left(2^{N}\right)$ \\
\hline MAG & Gustafsson [8] & 2002 & $\Omega\left(2^{N}\right)$ \\
\hline- & Bernstein [12] & 1986 & $\mathrm{O}\left(2^{N}\right)$ \\
\hline Hcub & Voronenko [6] & 2007 & $O\left(N^{6}\right)$ \\
\hline BHM & Dempster [13] & 1995 & $O\left(N^{4}\right)$ \\
\hline- & Lefèvre [4] & 2001 & $O\left(N^{3}\right)$ \\
\hline DBNS & Dimitrov [3] & 2007 & $O(N)$ \\
\hline CSD & Avizienis [2] & 1961 & $O(N)$ \\
\hline
\end{tabular}

The central point of this work is the minimization of the total number of additions. Based on radix $-2^{r}$ signed-digit number system [14] [15], a new Redundant Radix- $2^{r}$ Recoding (R3) is proposed as an alternative to existing heuristics. Applied to the particular case of radix $-2^{8}$ with $N=64$, a saving of $29.10 \%$ is achieved over CSD, which yields to much less power consumption and more speed. In addition, the new recoding shows high aptitude for common subexpression elimination, which makes it a good candidate for MCM.

The paper is organized as follows. Section I outlines the necessity of a linear runtime heuristic with a high compression ratio to handle large bit-size constants. Section II introduces the new R3 algorithm, while Section III compares the results to CSD and DBNS recodings. Finally, Section IV provides some concluding remarks and suggestions for future work.

\section{NEW REDUNDANT RADIX-2 ${ }^{r}$ ALGORITHM (R3) FOR MULTIPLICATION BY A $N$-BIT CONSTANT}

A $N$-bit $C$ constant is expressed in radix- $2^{r}$ as follows:

$$
\begin{aligned}
C & =\sum_{j=0}^{(N / r)-1}\left(c_{r j-1}+2^{0} c_{r j}+2^{1} c_{r j+1}+2^{2} c_{r j+2}+\cdots+2^{r-2} c_{r j+r-2}-2^{r-1} c_{r j+r-1}\right) \times 2^{r j} \\
& =\sum_{j=0}^{(N / r)-1} Q_{j} \times 2^{r j}
\end{aligned}
$$

where $\quad c_{-1}=0$ and $r \in \mathrm{N}^{*}$. For simplicity purposes and without loss of generality, we assume that $r$ is a divider of $N$. 
In eq. (1), the two's complement representation of $C$ constant is split into $N / r$ two's complement slices $\left(Q_{j}\right)$, each of $r+1$ bit length. Each pair of two contiguous slices has one overlapping bit. To eq. (1), corresponds a digit-set $D\left(2^{r}\right)$ such as

$$
Q_{j} \in D\left(2^{r}\right)=\left\{-2^{r-1},-2^{r-1}+1, \ldots,-1,0,1, \ldots, 2^{r-1}-1,2^{r-1}\right\} .
$$

The sign of $Q_{j}$ term is given by $c_{r j+r-1}$ bit, and $\left|Q_{j}\right|=2^{k_{j}} \times m_{j}$, with $k_{j} \in\{0,1,2, \ldots, r-1\}$ and $m_{j} \in O M\left(2^{r}\right)=\left\{1,3,5, \ldots, 2^{r-1}-1\right\} \cup\{0\}$. $O M\left(2^{r}\right)$ represents the required set of odd-multiples in radix- $2^{r}$ recoding, with $\left|O M\left(2^{r}\right)\right|=2^{r-2}$. Finally, $C$ can be expressed as follows: $\quad C=\sum_{j=0}^{(N / r)^{-1}}(-1)^{c_{r j+r-1}} \times\left(m_{j} \times 2^{k_{j}}\right) \times 2^{r j}$.

Equation (2) is not redundant since for each $C$ constant corresponds a unique representation $\left(m_{j}\right)$. To make the solution space larger in order to select a less adder-consuming representation of $C$, the recoding must be redundant. To achieve such a goal, we announce the following theorem:

Theorem 1. In radix $-2^{r},\left|Q_{j}\right|=\left[A_{j} \times 2^{p}+(-1)^{e} \times B_{j} \times 2^{h}\right]$, where: $A_{j}, B_{j} \in\left\{0,1,3,5, \ldots .2^{(r / 2)-1}-1\right\} ; p \in\left\{0,1,2, \ldots, 2^{r-1}-1\right\} ; h \in\left\{0,1,2, \ldots, 2^{(r / 2)-1}-1\right\}$, and $e \in\{0,1\}$.

The proof of the above theorem is based on our Theorem (1) described in [16][17]. Note that different notations for $\left|Q_{j}\right|$ are possible. For instance: $37=1 \times 2^{5}+5 \times 2^{0}$ or $37=5 \times 2^{3}-3 \times 2^{0}$. We illustrate the idea for $r=8$, where $0 \leq|Q j| \leq 128$. Equation (2) becomes: $C=\sum_{j=0}^{(N / 8)-1}\left(A_{j} \times 2^{p}+(-1)^{e} \times B_{j} \times 2^{h}\right) \times(-1)^{c_{8 j+7}} \times 2^{8 j}$

$$
=\sum_{j=0}^{(N / 8)-1}\left(Z_{1}+Z_{2}\right)_{j} \times(-1)^{c_{8 j+7}} \times 2^{8 j}
$$

where $\quad Z_{1}=A_{j} \times 2^{p} \quad ; \quad Z_{2}=(-1)^{\mathrm{e}} \times B_{j} \times 2^{h} \quad ; A_{j}, B_{j} \in\{0,1,3,5,7\}$; $p \in\{0,1,2, \ldots, 7\} ; h \in\{0,1,2,3\} ;$ and $e \in\{0,1\}$.

Note that $\left|Q_{j}\right|=\left(Z_{1}+Z_{2}\right)_{j}$. The partitioning of $C$ constant according to eq. (3) is depicted in Fig. 1, while the recodings of odd and even $\left|Q_{j}\right|$ digits are separately denoted in Table II.

The product $C \times X$ becomes:

$$
C \times X=\sum_{j=0}^{(N / 8)-1}\left[\left(A_{j} \times X\right) \times 2^{p}+(-1)^{e} \times\left(B_{j} \times X\right) \times 2^{h}\right] \times(-1)^{c_{8 j+7}} \times 2^{8 j}
$$

Note that when $A_{j}, B_{j} \in\{3,5,7\}$, one extra adder is needed since for instance: $3 \times X=2 \times X+X$.

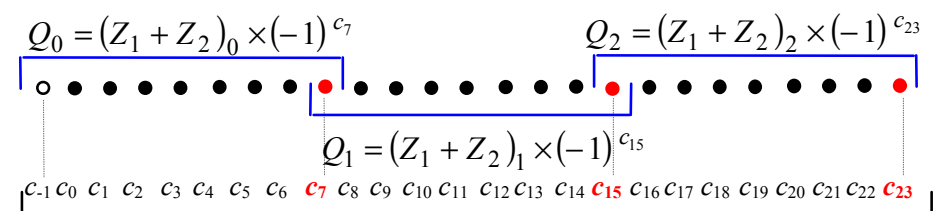

$$
C=Q_{0} \times 2^{0}+Q_{1} \times 2^{8}+Q_{2} \times 2^{16}
$$

\begin{tabular}{|c|c|c|c|c|c|}
\hline Odd $\left|Q_{j}\right|$ & $Z_{1}=A_{j} \times 2^{p}$ & $Z_{2}=(-1)^{\mathrm{e}} \times B_{j} \times 2^{h}$ & $\left(Z_{1}+Z_{2}\right)_{j}$ & $\overline{E \text { Even }\left|Q_{j}\right|}$ & $\left(Z_{1}+Z_{2}\right)$ \\
\hline 1 & $1 \times 2^{0}$ & $0 \times 2^{0}$ & $\mathrm{U}_{1}$ & 2 & $2^{1} \times U_{1}$ \\
\hline 3 & $3 \times 2^{0}$ & $0 \times 2^{0}$ & $\mathrm{U}_{3}$ & 4 & $2^{2} \times U_{1}$ \\
\hline 5 & $5 \times 2^{0}$ & $0 \times 2^{0}$ & $\mathrm{U}_{5}$ & 6 & $2^{1} \times U_{3}$ \\
\hline 7 & $7 \times 2^{0}$ & $0 \times 2^{0}$ & $\mathrm{U}_{7}$ & 8 & $2^{3} \times U_{1}$ \\
\hline 9 & $1 \times 2^{3}$ & $1 \times 2^{0}$ & $\mathrm{U}_{9}$ & 10 & $2^{1} \times U_{5}$ \\
\hline 11 & $3 \times 2^{2}$ & $-1 \times 2^{0}$ & $\mathrm{U}_{11}$ & 12 & $2^{2} \times \mathrm{U}_{3}$ \\
\hline 13 & $3 \times 2^{2}$ & $1 \times 2^{0}$ & $\mathrm{U}_{13}$ & 14 & $2^{1} \times U_{7}$ \\
\hline 15 & $1 \times 2^{4}$ & $-1 \times 2^{0}$ & $\mathrm{U}_{15}$ & 16 & $2^{4} \times U_{1}$ \\
\hline 17 & $1 \times 2^{4}$ & $1 \times 2^{0}$ & $\mathrm{U}_{17}$ & 18 & $2^{1} \times U_{9}$ \\
\hline 19 & $5 \times 2^{2}$ & $-1 \times 2^{0}$ & $\overline{\mathrm{U}_{19}}$ & 20 & $2^{2} \times U_{5}$ \\
\hline 21 & $5 \times 2^{2}$ & $1 \times 2^{0}$ & $\mathrm{U}_{21}$ & 22 & $2^{1} \times U_{11}$ \\
\hline 23 & $3 \times 2^{3}$ & $-1 \times 2^{0}$ & $\mathrm{U}_{23}$ & 24 & $2^{3} \times \mathrm{U}_{3}$ \\
\hline 25 & $3 \times 2^{3}$ & $1 \times 2^{0}$ & $\mathrm{U}_{25}$ & 26 & $2^{1} \times U_{13}$ \\
\hline 27 & $7 \times 2^{2}$ & $-1 \times 2^{0}$ & $\mathrm{U}_{27}$ & 28 & $2^{2} \times U_{7}$ \\
\hline 29 & $7 \times 2^{2}$ & $1 \times 2^{0}$ & $\mathrm{U}_{29}$ & 30 & $2^{1} \times U_{15}$ \\
\hline 31 & $1 \times 2^{5}$ & $-1 \times 2^{0}$ & $\mathrm{U}_{31}$ & 32 & $2^{5} \times U_{1}$ \\
\hline 33 & $1 \times 2^{5}$ & $1 \times 2^{0}$ & $\mathrm{U}_{33}$ & 34 & $2^{1} \times U_{17}$ \\
\hline 35 & $1 \times 2^{5}$ & $3 \times 2^{0}$ & $\mathrm{U}_{35}$ & 36 & $2^{2} \times U_{9}$ \\
\hline 37 & $1 \times 2^{5}$ & $5 \times 2^{0}$ & $\mathrm{U}_{37}$ & 38 & $2^{1} \times U_{19}$ \\
\hline 39 & $5 \times 2^{3}$ & $-1 \times 2^{0}$ & $\mathrm{U}_{39}$ & 40 & $2^{3} \times \mathrm{U}_{5}$ \\
\hline 41 & $5 \times 2^{3}$ & $1 \times 2^{0}$ & $\mathrm{U}_{41}$ & 42 & $2^{1} \times U_{21}$ \\
\hline 43 & $5 \times 2^{3}$ & $3 \times 2^{0}$ & $\mathrm{U}_{43}$ & 44 & $2^{2} \times U_{11}$ \\
\hline 45 & $3 \times 2^{4}$ & $-3 \times 2^{0}$ & $\mathrm{U}_{45}$ & 46 & $2^{1} \times U_{23}$ \\
\hline 47 & $3 \times 2^{4}$ & $-1 \times 2^{0}$ & $\mathrm{U}_{47}$ & 48 & $2^{4} \times U_{3}$ \\
\hline 49 & $3 \times 2^{4}$ & $1 \times 2^{0}$ & $\mathrm{U}_{49}$ & 50 & $2^{1} \times U_{25}$ \\
\hline 51 & $3 \times 2^{4}$ & $3 \times 2^{0}$ & $\mathrm{U}_{51}$ & 52 & $2^{2} \times U_{13}$ \\
\hline 53 & $3 \times 2^{4}$ & $5 \times 2^{0}$ & $\mathrm{U}_{53}$ & 54 & $2^{1} \times U_{27}$ \\
\hline 55 & $7 \times 2^{3}$ & $-1 \times 2^{0}$ & $\mathrm{U}_{55}$ & 56 & $2^{3} \times \mathrm{U}_{7}$ \\
\hline 57 & $7 \times 2^{3}$ & $1 \times 2^{0}$ & $\mathrm{U}_{57}$ & 58 & $2^{1} \times U_{29}$ \\
\hline 59 & $1 \times 2^{6}$ & $-5 \times 2^{0}$ & $\mathrm{U}_{59}$ & 60 & $2^{4} \times U_{15}$ \\
\hline 61 & $1 \times 2^{6}$ & $-3 \times 2^{0}$ & $\mathrm{U}_{61}$ & 62 & $2^{1} \times U_{31}$ \\
\hline 63 & $1 \times 2^{6}$ & $-1 \times 2^{0}$ & $\mathrm{U}_{63}$ & 64 & $2^{6} \times \mathrm{U}_{1}$ \\
\hline 65 & $1 \times 2^{6}$ & $1 \times 2^{0}$ & $\mathrm{U}_{65}$ & 66 & $2^{1} \times U_{33}$ \\
\hline 67 & $1 \times 2^{6}$ & $3 \times 2^{0}$ & $\mathrm{U}_{67}$ & 68 & $2^{2} \times U_{17}$ \\
\hline 69 & $1 \times 2^{6}$ & $5 \times 2^{0}$ & $\mathrm{U}_{69}$ & 70 & $2^{1} \times U_{35}$ \\
\hline 71 & $1 \times 2^{6}$ & $7 \times 2^{0}$ & $\mathrm{U}_{71}$ & 72 & $2^{3} \times U_{9}$ \\
\hline 73 & $5 \times 2^{4}$ & $-7 \times 2^{0}$ & $\mathrm{U}_{73}$ & 74 & $2^{1} \times U_{37}$ \\
\hline 75 & $5 \times 2^{4}$ & $-5 \times 2^{0}$ & $\mathrm{U}_{75}$ & 76 & $2^{4} \times U_{19}$ \\
\hline 77 & $5 \times 2^{4}$ & $-3 \times 2^{0}$ & $\mathrm{U}_{77}$ & 78 & $2^{1} \times U_{39}$ \\
\hline 79 & $5 \times 2^{4}$ & $-1 \times 2^{0}$ & $\mathrm{U}_{79}$ & 80 & $2^{4} \times U_{5}$ \\
\hline 81 & $5 \times 2^{4}$ & $1 \times 2^{0}$ & $\mathrm{U}_{81}$ & 82 & $2^{1} \times U_{41}$ \\
\hline 83 & $5 \times 2^{4}$ & $3 \times 2^{0}$ & $\mathrm{U}_{83}$ & 84 & $2^{2} \times U_{21}$ \\
\hline 85 & $5 \times 2^{4}$ & $5 \times 2^{0}$ & $\mathrm{U}_{85}$ & 86 & $2^{1} \times U_{43}$ \\
\hline 87 & $5 \times 2^{4}$ & $7 \times 2^{0}$ & $\mathrm{U}_{87}$ & 88 & $2^{3} \times U_{11}$ \\
\hline 89 & $3 \times 2^{5}$ & $-7 \times 2^{0}$ & $\mathrm{U}_{89}$ & 90 & $2^{1} \times \mathrm{U}_{45}$ \\
\hline 91 & $3 \times 2^{5}$ & $-5 \times 2^{0}$ & $\mathrm{U}_{91}$ & 92 & $2^{2} \times U_{23}$ \\
\hline 93 & $3 \times 2^{5}$ & $-3 \times 2^{0}$ & $\mathrm{U}_{93}$ & 94 & $2^{1} \times U_{47}$ \\
\hline 95 & $3 \times 2^{5}$ & $-1 \times 2^{0}$ & $\mathrm{U}_{95}$ & 96 & $2^{5} \times \mathrm{U}_{3}$ \\
\hline 97 & $3 \times 2^{5}$ & $1 \times 2^{0}$ & $\mathrm{U}_{97}$ & 98 & $2^{1} \times U_{49}$ \\
\hline 99 & $3 \times 2^{5}$ & $3 \times 2^{0}$ & $\mathrm{U}_{99}$ & 100 & $2^{2} \times U_{25}$ \\
\hline 101 & $3 \times 2^{5}$ & $5 \times 2^{0}$ & $\mathrm{U}_{101}$ & 102 & $2^{1} \times U_{51}$ \\
\hline 103 & $3 \times 2^{5}$ & $7 \times 2^{0}$ & $\mathrm{U}_{103}$ & 104 & $2^{3} \times \mathrm{U}_{13}$ \\
\hline 105 & $7 \times 2^{4}$ & $-7 \times 2^{0}$ & $\mathrm{U}_{105}$ & 106 & $2^{1} \times U_{53}$ \\
\hline 107 & $7 \times 2^{4}$ & $-5 \times 2^{0}$ & $\mathrm{U}_{107}$ & 108 & $2^{2} \times U_{27}$ \\
\hline 109 & $7 \times 2^{4}$ & $-3 \times 2^{0}$ & $\mathrm{U}_{109}$ & 110 & $2^{1} \times U_{55}$ \\
\hline 111 & $7 \times 2^{4}$ & $-1 \times 2^{0}$ & $\mathrm{U}_{111}$ & 112 & $2^{4} \times U_{7}$ \\
\hline 113 & $7 \times 2^{4}$ & $1 \times 2^{0}$ & $\mathrm{U}_{113}$ & 114 & $2^{1} \times U_{57}$ \\
\hline 115 & $7 \times 2^{4}$ & $3 \times 2^{0}$ & $U_{115}$ & 116 & $2^{3} \times U_{29}$ \\
\hline 117 & $7 \times 2^{4}$ & $5 \times 2^{0}$ & $\mathrm{U}_{117}$ & 118 & $2^{1} \times U_{59}$ \\
\hline 119 & $7 \times 2^{4}$ & $7 \times 2^{0}$ & $\mathrm{U}_{119}$ & 120 & $2^{3} \times \mathrm{U}_{15}$ \\
\hline 121 & $1 \times 2^{7}$ & $-7 \times 2^{0}$ & $\mathrm{U}_{121}$ & 122 & $2^{1} \times \mathrm{U}_{61}$ \\
\hline 123 & $1 \times 2^{7}$ & $-5 \times 2^{0}$ & $\mathrm{U}_{123}$ & 124 & $2^{2} \times U_{31}$ \\
\hline 125 & $1 \times 2^{7}$ & $-3 \times 2^{0}$ & $\mathrm{U}_{125}$ & 126 & $2^{1} \times U_{63}$ \\
\hline 127 & $1 \times 2^{7}$ & $-1 \times 2^{0}$ & $\mathrm{U}_{127}$ & 128 & $2^{7} \times \mathrm{U}_{1}$ \\
\hline
\end{tabular}

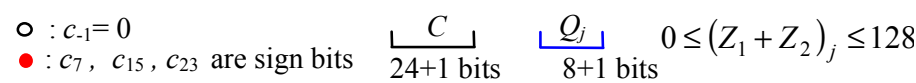

Figure 1. Partitioning of a 24-bit $C$ constant using R3 algorithm.
TABLE II: ODD AND EVEN $\left|Q_{\jmath}\right|$ DIGIT RECODING USING R3 ALGORITHM 
Our recoding is highly redundant, i.e., each $\left|Q_{j}\right|$ may have several notations in $Z_{1}$ and $Z_{2}$ digits. We fully exploited this property to minimize the number of adders using a $\mathrm{C}$-program which exhaustively explores for each odd $\left|Q_{j}\right|$, all possible notations and selects the least adder consumer combination according to the following priority order: $\left(A_{j}, B_{j}\right)=\left(A_{j}, 0\right) ;\left(A_{j}\right.$, $\left.B_{j}\right)=(1,1) ;\left(Z_{1}, Z_{2}\right)=\left(1 \times 2^{7}, Z_{2}\right)$; and finally $\left(Z_{1}, Z_{2}\right)=\left(Z_{1}, 1 \times 2^{0}\right)$. These two latter couples allow the following simplification:

$$
\ldots+\left[\left(1 \times 2^{7}+Z_{2}\right) \times 2^{8 j}\right\rfloor+\left\lfloor\left(Z_{1}-1 \times 2^{0}\right) \times 2^{8 j+8}\right\rfloor+\ldots=\ldots+\left\lfloor Z_{2} \times 2^{8 j}-2\right\rfloor+\left\lfloor Z_{1} \times 2^{8 j+8}\right\rfloor+\ldots
$$

In case none of those cases is encountered, C-program pursues in the following priority order: $(A j, B j)=(1,3)$ or $(3,1)$; $(A j, B j)=(3,3) ;(A j, B j)=(1,5)$ or $(5,1) ;(A j, B j)=(5,5) ;(A j$, $B j)=(1,7)$ or $(7,1) ;(A j, B j)=(7,7) ;(A j, B j)=(3,5)$ or $(5,3)$; $(A j, B j)=(3,7)$ or $(7,3) ;(A j, B j)=(5,7)$ or $(7,5)$. This order maximizes the occurrences of 1 , then of 3 , and minimizes those of 5 and 7 in $|Q j|$ digits, which will more likely reduce the number of adders in the whole $C$ recoding. Furthermore, we perform common $\mathrm{U}_{\mathrm{k}}$ digit elimination as an ultimate optimization step. Only odd $|Q j|$ digits are optimized. Optimized even digits are directly derived from odd ones using shift operations as indicated in Table II.

To illustrate the idea, the product $\mathrm{P}=23453 \times X$ is first computed in CSD and then in R3. It gives:

$\mathrm{P}_{\mathrm{CSD}}=2^{15} \times X-2^{13} \times X-2^{10} \times X-2^{7} \times X+2^{5} \times X-2^{2} \times X+X ;$ $\mathrm{P}_{\mathrm{R} 3}=2^{8} \times\left(2^{5} \times \mathrm{U}_{3}-2^{2} \times \mathrm{U}_{1}\right)-\left(2^{5} \times \mathrm{U}_{3}+\mathrm{U}_{3}\right) ; \mathrm{U}_{1}=X$ and $\mathrm{U}_{3}=2 \times X+X$.

$\mathrm{P}_{\mathrm{CSD}}$ requires 6 operations, while $\mathrm{P}_{\mathrm{R} 3}$ needs only 4 . Note that the naïve add-and-shift algorithm would have required 9 operations. We assume that addition and subtraction have the same area/speed cost, and that shift is costless since it can be realized without any gates using hard wiring. Note that in R3 there is no overflow risk since the shift span is fully controlled.

\section{RESULT COMPARISON}

In equation (4), there are $N / 8$ iterations. Each iteration generates a maximum of 2 partial products (PP). Thus, the maximal number of PP is $N / 4$. A maximum of 3 supplementary adders are necessary in case $3 \times \mathrm{X}, 5 \times \mathrm{X}$, and $7 \times \mathrm{X}$ are all invoked at the same time in the recoding. Therefore, the maximal number of additions per coefficient $(U p b)$ is bounded by $(N / 4)+2$. As for the average number of additions $(A v g)$, it has been exhaustively calculated for $C$ values varying from 0 to $2^{N}-1$, for $N=8,16,24$, and 32 . But for $N=64$, we calculated the average using $10^{5}, 10^{6}, 10^{9}$ and $10^{10}$ uniformly distributed random $C$ values. While the difference between the four obtained results is insignificant $\left(<10^{-3}\right)$, the average decreases as the number of $C$ values increases, and converges to 14.4932 additions. Results are reported in Table III. For $N=64$, R3 uses $29.10 \%$ less additions than CSD. The saving seems to grow linearly for low values of $N$. It will asymptotically converge to an upper limit which is unknown for the time being.

Regarding computation-time complexity, it is linearly proportional to $N$ as shown by eq. (4). As for the storage complexity, a look-up table with 128 entries is required, which is insignificant.
Concerning DBNS, Dimitrov [3] calculated average and upper-bound values from $10^{5}$ uniformly distributed random constants, for 32 and 64 bits only (Table IV). Note that DBNS upper-bounds will be higher if the worst cases are not attained by the pattern of $10^{5}$ constants.

TABLE III: R3 VERSUS CSD : AVERAGE NUMBER OF ADDITIONS $(A v g)$ AND UPPER BOUND $(U p b)$

\begin{tabular}{|c|c|c|c|c|c|}
\hline \hline \multirow{2}{*}{$\begin{array}{c}\text { Constant } \\
\text { Bit-width } N\end{array}$} & \multicolumn{2}{|c|}{ CSD } & \multicolumn{2}{c|}{$\mathrm{R} 3$} & \multirow{2}{*}{$\begin{array}{l}\text { Saving } \\
(A v g, \%)\end{array}$} \\
\cline { 2 - 5 } & $A v g$ & $U p b$ & $A v g$ & $U p b$ & 3.5119 \\
\hline 8 & 1.7882 & 4 & 1.7254 & 3 & 3.6386 \\
\hline 16 & 4.4445 & 8 & 4.1050 & 6 & 7.6226 \\
\hline 24 & 7.1111 & 12 & 6.2846 & 8 & 11.646 \\
\hline 32 & 9.7777 & 16 & 8.3194 & 10 & 14.9145 \\
\hline 64 & 20.4444 & 32 & $14.4932^{*}$ & 18 & 29.1091 \\
\hline \hline
\end{tabular}

*: Obtained from $10^{10}$ uniformly distributed random $C$ values.

TABLE IV: R3 VERSUS DBNS : AVERAGE NUMBER OF ADDITIONS (Avg) AND UPPER BOUND (Upb)

\begin{tabular}{|c|c|c|c|c|c|}
\hline \multirow{2}{*}{$\begin{array}{c}\text { Constant } \\
\text { Bit-width } N\end{array}$} & \multicolumn{2}{|c|}{ DBNS [3] } & \multicolumn{2}{|c|}{ R3 } & \multirow{2}{*}{$\begin{array}{l}\text { Saving } \\
(\text { Avg, \%) }\end{array}$} \\
\hline & Avg & Upb & Avg & Upb & \\
\hline 32 & $\approx 9.05^{+*}$ & $13^{*}$ & 8.3194 & 10 & 8.0729 \\
\hline 64 & $16.2151^{*}$ & $21^{*}$ & 14.4932 & 18 & 10.6191 \\
\hline
\end{tabular}

+: Taken from Fig.1 in [3]; *: Obtained from $10^{5}$ uniformly distributed random constants.

Another performance indicator of the recoding is the smallest value that requires $q$ additions, for $q$ varying from 1 to the upper-bound of the recoding. Table $\mathrm{V}$ summarizes this information for 32-bit constant. Note that starting from $q=7$, higher values are provided by R3 algorithm.

TABLE V: R3 VERSUS CSD : SMALLEST VALUES
FOR 32-BIT CONSTANT
\begin{tabular}{|c|c|c|}
\hline \hline $\begin{array}{c}\text { Number of } \\
\text { Additions }(q)\end{array}$ & CSD & R3 \\
\hline 1 & 3 & 3 \\
\hline 2 & 11 & 11 \\
\hline 3 & 43 & 43 \\
\hline 4 & 171 & 139 \\
\hline 5 & 683 & 651 \\
\hline 6 & 2731 & 2699 \\
\hline 7 & 10923 & 34971 \\
\hline 8 & 43691 & 559259 \\
\hline 9 & 174763 & 17336475 \\
\hline 10 & 699051 & 143163547 \\
\hline 11 & 2796203 & - \\
\hline 12 & 11184811 & - \\
\hline 13 & 44739243 & - \\
\hline 14 & 178956971 & - \\
\hline 15 & 715827883 & - \\
\hline \hline
\end{tabular}

Predictability in addition-number ( $U p b$ and $A v g$ ) and runtime/storage requirements informs on the heuristic capabilities and limitations. $U p b$ denotes exactly the length of the critical-path formed by successive additions, while Avg gives an idea on the compression performance of the heuristic. On the other hand, runtime/storage complexity helps to decide whether the use of the heuristic is appropriate with regard to a constant bit-width $(N)$. While this latter is known for all heuristics (Table I), addition complexity is unknown for most of them [1][11]. Pinch was the first to set an asymptotic 
complexity $O(N / \log (N))$ for $U p b$ [18]. Better, based on DBNS arithmetic [19], Dimitrov [20] gave a rough evaluation of the hidden constant $(\alpha)$ in the big $O$-notation as being $1 \leq \alpha \leq 2$. Only CSD and R3 do have exact analytic expressions for addition complexity (only $U p b$ for R3). For the all remaining heuristics, no addition complexity does exist. This is a real handicap as there is no visibility on how the heuristic evolves with respect to $N$, unless to exhaustively calculate $A v g$ (Fig. 2) and $U p b$, but this is still limited to low values of $(N \leq 32)$ as an excessive compute power is required. Though heuristics of Fig. 2 exhibits higher compression ratios than R3 for $N>16$, some values of Table VI are not only greater than the ones provided by R3, but also equal or even greater than $U p b$ of R3. For $N \geq 128$, only Lefèvre algorithm remains practical $O\left(N^{3}\right)$, because even when neglecting the hidden constant $\alpha$ in $O\left(N^{6}\right)$, Hcub requires more than 4398 billions of iterations. Another serious drawback of non-recoding heuristics is the overflow risk because of uncontrolled shift spans [3]. Such a problem never occurs in digit-recoding heuristics: CSD, DBNS and R3.

It becomes now clear why despite the large number of existing heuristics; CSD is not only used in designing the vast majority of LTI systems [10], but incorporated in most of advanced synthesis tool as well, such as in Synopsys Design Compiler Ultra [10][21].

\section{CONCLUSION AND FUTURE WORK}

An efficient alternative (R3) to the most commonly used heuristic (CSD) has been proposed. Instead CSD, the use of R3 in designing LTI systems leads to much less power consumption and more speed. A pending issue is to determine the analytic expression of the average number of additions ( $A v g$ ) needed by R3 with regard to constant bit-width $N$.

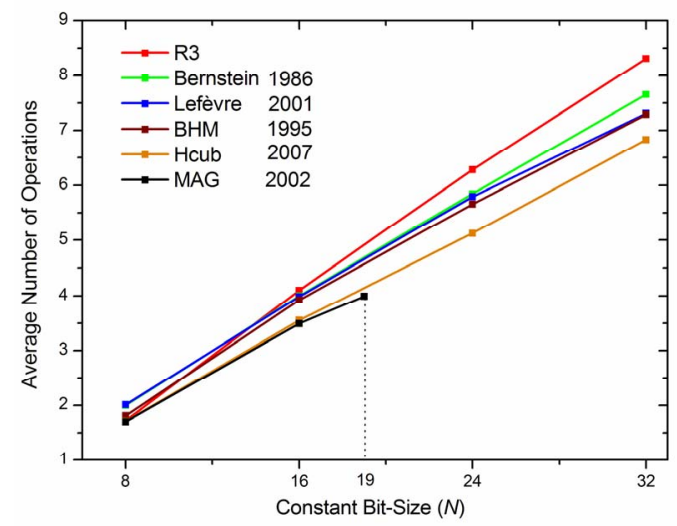

Figure 2. Comparison of R3 with non-recoding heuristics based on average number of additions $(A v g)$

TABLE VI: NUMBER OF ADDERS: SOME PECULIARITIES

\begin{tabular}{|l|c|c|c|c|}
\hline \hline \multirow{3}{*}{ Algorithm } & \multicolumn{4}{|c|}{ Hexadecimal Values } \\
\cline { 2 - 5 } & $\begin{array}{c}(84 \mathrm{AB} 5)_{\mathrm{H}} \\
N=20\end{array}$ & $\begin{array}{c}(595959)_{\mathrm{H}} \\
N=24\end{array}$ & $\begin{array}{c}(64 \mathrm{AB} 55)_{\mathrm{H}} \\
N=24\end{array}$ & $\begin{array}{c}(5959595 \mathrm{~B})_{\mathrm{H}} \\
N=32\end{array}$ \\
\hline Bernstein [12] & $8^{\mathrm{G}}$ & 7 & 7 & $\underline{8}$ \\
\hline Hcub* $[6]^{*}$ & 6 & $8^{\mathrm{E}}$ & $9^{\mathrm{G}}$ & - \\
\hline BHM $^{*}[13]$ & 5 & 7 & 7 & - \\
\hline Lefèvre [4] & $\underline{4}$ & $8^{\mathrm{E}}$ & $\underline{6}$ & $11^{\mathrm{G}}$ \\
\hline R3 & $\underline{4}$ & $\underline{5}$ & $\underline{6}$ & $\underline{8}$ \\
\hline
\end{tabular}

*: Limited to 26 bits; $\mathrm{x}$ : Lowest number of additions ; $N$ : Constant bit-size; E: Equal to $U p b$ of R3; G: Greater than $U p b$ of R3; $U p b$ of R3= $(N / 4)+2$

\section{REFERENCES}

[1] J. Thong and N. Nicolici, "An optimal and practical approach to single constant multiplication," IEEE Trans. on Computer-Aided Design of Integrated Circuits and Systems, vol. 30, no. 9, pp. 1373-1386, September 2011.

[2] A. Avizienis, "Signed-digit number representation for fast parallel arithmetic,". IRE Trans. on Electronic Computers, vol. EC-10, No. 3, pp. 389-400, September 1961.

[3] V.S. Dimitrov, L. Imbert, and A. Zakaluzny, "Multiplication by a Constant is Sublinear," Proceedings of the 18th IEEE Symposium on Computer Arithmetic (ARITH'18), pp. 261-268, June 2007.

[4] V. Lefèvre, "Multiplication by an Integer Constant," INRIA Research Report, No. 4192, Lyon, France, May 2001.

[5] N. Boullis and A. Tisserand, "Some Optimizations of Hardware Multiplication by Constant Matrices," IEEE Trans. on Computers (TC), vol. 54, No. 10, pp. 1271-1282, October 2005.

[6] Y. Voronenko and M. Püschel, "Multiplierless Multiple Constant Multiplication," ACM Trans. on Algorithms (TALG), vol. 3, No. 2, Artcle 11, pp. 1-38, May 2007.

[7] A. Dempster and M. Macleod, "Using Signed-Digit Representations to Design Single Integer Multipliers Using Subexpression Elimination," Proceedings of the IEEE International Symp. on Circuits and Systems (ISCAS), vol. 3, pp. III-165-168, Vancouver, Canada, May 2004.

[8] O. Gustafsson, A.G. Dempster, and L. Wanhammar, "Extended Results for Minimum-Adder Constant Integer Multipliers," Proceedings of the IEEE International Symposium on Circuits and Systems (ISCAS), vol. 1, pp. I-73 I-76, Scottsdale Arizona, USA, May 2002.

[9] F. de Dinechin, "Multiplication by Rational Constant," IEEE Trans. on Circuits and Systems II: Express Brief, vol. 59, No. 2, pp. 98-102, February 2012.

[10] R. Kastner, A. Hosangadi, and F. Fallah, "Arithmetic Optimization Techniques for Hardware and Software Design," Cambridge University Press, ISBN-13 978-0-521-88099-2, (C) 2010.

[11] O. Gustafsson, "Lower Bounds for Constant Multiplication Problems," IEEE Trans. on Circuits and Systems II: Express Brief, vol. 54, No. 11, pp. 974-978, November 2007.

[12] R.L. Bernstein, "Multiplication by Integer Constant," Software Practice and Experience 16, 7, pp. 641-652, 1986.

[13] A.G. Dempster and M.D. Macleod, "Use of Minimum Adder Multiplier Blocks in FIR Digital Filters," IEEE Trans. on Circuits and Systems-II: Analog and Digital Signal Processing 42, 9, pp. 569-567, 1995.

[14] S. Homayoon and A. Gupta, "A Generalized Multibit Recoding of Two's Complement Binary Numbers and its Proof with Application in Multiplier Implementation,” IEEE Trans. on Computers (TC), vol. 39, $\mathrm{N}^{\circ} 8$, August 1990.

[15] P.M. Seidel, L. D. McFearin, and D.W. Matula, "Secondary Radix Recodings for Higher Radix Multipliers," IEEE Trans. on Computers (TC), vol. 54, N², February 2005.

[16] A.K. Oudjida, N. Chaillet, A. Liacha, and M.L. Berrandjia "New HighSpeed and Low-Power Radix-2 ${ }^{\mathrm{r}}$ Multiplication Algorithms," Proceedings of the 11th edition of IEEE-FTFC Low-Voltage LowPower Conference, ISSN: 978-1-4673-0821-2/12, Paris, June 2012.

[17] A.K. Oudjida, N. Chaillet, A. Liacha, and M.L. Berrandjia, "A New Recursive Multibit Recoding Algorithm for High-Speed and Low-Power Multiplier," Journal of Low Power Electronics (JOLPE), vol. 8, $\mathrm{N}^{\circ} 5$, pp. 579-594, ISSN 1546-1998, American Scientific Publishers (ASP), December 2012.

[18] R. G. E. Pinch, "Asymptotic Upper Bound for Multiplier Design," Electronics Letters, vol. 32, N 5, pp. 420-421, February 1996.

[19] V.S. Dimitrov, G.A. Jullien, and W.C. Miller, "Theory and Applications of the Double-Base Number System," IEEE Trans. on Computers (TC), vol. 48, No. 10, pp. 1098-1106, October 1999.

[20] V.S. Dimitrov, K.U. Järvinen, and J. adikari, “Area Efficient Multipliers Based on Multiple-Radix Representations," IEEE Trans. on Computers (TC), vol. 60, N², pp 189-201, February 2011.

[21] Synopsys Datasheet, Design Compiler Ultra-Design Compiler® at its Best. Available at: www.synopsys.com. 